\title{
A model for pseudo-Dirac neutrinos: leptogenesis and ultra-high energy neutrinos
}

\author{
Y.H. Ahn, ${ }^{a}$ Sin Kyu Kang ${ }^{b}$ and C.S. Kim ${ }^{c}$ \\ ${ }^{a}$ Center for Theoretical Physics of the Universe, Institute for Basic Science (IBS), \\ Daejeon, 34051 Korea \\ ${ }^{b}$ Insitute for Convergence Fundamental Study, School of Liberal Arts, Seoul-Tech., \\ Seoul, 01811 Korea \\ ${ }^{c}$ Dept. of Physics and IPAP, Yonsei University, \\ Seoul, 120-749 Korea \\ E-mail: yhahn@ibs.re.kr, skkang@snut.ac.kr, cskim@yonsei.ac.kr
}

ABSTRACT: We propose a model where sterile neutrinos are introduced to make light neutrinos to be pseudo-Dirac particles. It is shown how tiny mass splitting necessary for realizing pseudo-Dirac neutrinos can be achieved. Within the model, we show how leptogenesis can be successfully generated. Motivated by the recent observation of very high energy neutrino events at IceCube, we study a possibility to observe the effects of the pseudo-Dirac property of neutrinos by performing astronomical-scale baseline experiments to uncover the oscillation effects of very tiny mass splitting. We also discuss future prospect to observe the effects of the pseudo-Dirac property of neutrinos at high energy neutrino experiments.

Keywords: Beyond Standard Model, Neutrino Physics, Cosmology of Theories beyond the SM

ARXIV EPRINT: 1602.05276 


\section{Contents}

1 Introduction 1

2 A model for pseudo-Dirac neutrinos 2

3 Leptogenesis with pseudo-Dirac neutrinos 4

4 Probing pseudo-Dirac neutrinos at astronomical-scale experiments $\quad 8$

4.1 Results for the case of $\Delta m_{1,2,3}^{2}=\Delta m_{k}^{2} \quad 11$

$\begin{array}{lll}4.2 & \text { Results for the case of } \Delta m_{i}^{2} \gg \Delta m_{j}^{2} & 12\end{array}$

$\begin{array}{lll}5 & \text { Conclusion } & 15\end{array}$

\section{Introduction}

Sterile neutrino not only can be a good candidate for dark matter [1-21] but also play an essential role in achieving smallness of neutrino masses [22-24] and baryogenesis via leptogenesis [25]. The sterile neutrino states can mix with the active neutrinos and such admixtures contribute to various processes which are forbidden in the Standard Model (SM), and affect the interpretations of cosmological and astrophysical observations. Thus, the masses of the sterile neutrinos and their mixing with the active neutrinos are subject to various experimental bounds as well as cosmological and astrophysical constraints [26-33].

While we do not have any clue to decide whether neutrinos are Dirac or Majorana particles, here we would like to investigate for neutrinos to be pseudo-Dirac particles [34, 35]. There have been several literatures to study neutrino as a pseudo-Dirac particle [3647, 50]. Most of them have phenomenologically studied pseudo-Dirac neutrinos with very tiny mass splitting. In this work, we propose a model where sterile neutrinos are introduced to make light neutrinos to be pseudo-Dirac particles. We also show how tiny mass splitting necessary for realizing pseudo-Dirac neutrinos can be achieved. We also examine how leptogenesis can be successfully generated within the model.

From the phenomenological point of view, one of the most important questions must be how we can probe the pseudo-Dirac neutrinos. The magnitude of mass splittings for pseudo-Dirac neutrinos should be smaller than the solar, atmospheric and reactor neutrino mass scales, otherwise they should have affected neutrino oscillations for solar, atmospheric and terrestrial neutrinos. In order to investigate pseudo-Dirac neutrinos with very tiny mass splitting, we need to increase the propagation length of the neutrinos, and thus astrophysi$\mathrm{cal} /$ cosmic neutrinos detectable at neutrino telescope can provide us with the opportunity. In this work, we examine a possibility to observe the effects of the pseudo-Dirac property of neutrinos by performing astronomical-scale baseline experiments to uncover the oscillation effects of very tiny mass splitting. If the oscillation effects induced by pseudo-Dirac 
neutrinos with very high energy and long trajectory are prominent, then they may affect the observables detected at neutrino telescope. The neutrino flavor composition detected from the ultra-high energy neutrino experiments can serve as the observable to probe the effects of the pseudo-Dirac neutrinos [51-67].

Recently, IceCube experiments announced the observation of vey high energy neutrino events $[68,69]$. Analyzing the high energy neutrino events observed at IceCube, the trackto-shower ratio of the subset with energy above $60 \mathrm{TeV}$ has been studied in ref. [70]. They have shown that different production mechanisms for high energy neutrinos lead to different predictions of the ratio. Based on those results, we study how the oscillation effects induced by pseudo-Dirac neutrinos may affect the track-to-shower ratio. Given neutrino energy and mass splittings, the oscillation effects depend on neutrino trajectory in addition to neutrino mixing angles and CP phase. In our numerical analysis, we take the result of global fit to neutrino data for the input of neutrino mixing angles and CP phase. Thus, we examine how the oscillation peaks appear along with neutrino trajectory and discuss some implication on the numerical results.

This paper is organized as follows: in section 2, we describe a model which is an extension of the SM through the introduction of sterile neutrinos and show how pseudo-Dirac neutrinos can be realized. In section 3 , we examine how leptogenesis can be successfully generated in this model. In section 4 , we study how the pseudo-Dirac property of neutrinos can be probed through the results of high energy neutrino experiments. In section 5, we draw our conclusions.

\section{A model for pseudo-Dirac neutrinos}

In order to realize pseudo-Dirac neutrinos, let us consider the renormalizable Lagrangian given in the charged lepton basis as

$$
-\mathcal{L}=\frac{1}{2} \overline{N_{R}^{c}} M_{R} N_{R}+\bar{L} \tilde{\Phi} Y_{D} N_{R}+\bar{L} \tilde{\Phi} Y_{D S} S+\overline{S^{c}} \Psi Y_{S} N_{R}+\frac{1}{2} \overline{S^{c}} \mu S+\text { h.c. }
$$

where $L, N_{R}, S$ stand for SU $(2)_{L}$ left-handed lepton doublet, right-handed singlet and newly introduced singlet neutrinos, respectively, and $\tilde{\Phi} \equiv i \tau_{2} \Phi^{*} . M_{R}$ and $\mu$ are Majorana masses for the $N_{R}$ and $S$ fields, respectively. On top of the SM Higgs doublet $\Phi=\left(\phi^{+}, \phi^{0}\right)^{T}$, an $\mathrm{SU}(2)_{L}$ singlet scalar field $\Psi$ is introduced. Assigning quantum numbers $L: 1, N_{R}, S: 1$, $\Psi:-2$, and $\Phi: 0$ under the $\mathrm{U}(1)_{L}$ (or $\mathrm{U}(1)_{B-L}$ ) symmetry, the above Lagrangian is invariant under $\mathrm{U}(1)_{B-L}$ when $\mu, M_{R}=0$. So here the parameters $\mu, M_{R}$ reflect soft symmetry breaking of $\mathrm{U}(1)_{L}$. When the scalar field $\Psi$ attains a vacuum expectation value (VEV), it spontaneously breaks the $\mathrm{U}(1)_{L}$ (or $\mathrm{U}(1)_{B-L}$ ) symmetry, but does not break the electroweak gauge symmetry. Thus its VEV is not required to lie at the electroweak scale. Since the masses of Majorana neutrino $N_{R}$ are much larger than those of Dirac and light Majorana ones, we can integrate out the heavy Majorana neutrinos in the Lagrangian eq. (2.1), resulting in the following effective Lagrangian for neutrino sectors,

$$
\begin{aligned}
-\mathcal{L}_{\text {eff }}= & \overline{\nu_{L}} \phi^{0} Y_{D S} S-\frac{1}{2} \overline{\nu_{L}} \phi^{0} Y_{D} M_{R}^{-1} Y_{D}^{T} \phi^{0} \nu_{L}^{c}-\overline{\nu_{L}} \phi^{0} Y_{D} M_{R}^{-1} Y_{S}^{T} \Psi S \\
& -\frac{1}{2} \overline{S^{c}} \Psi Y_{S} M_{R}^{-1} Y_{S}^{T} \Psi S+\frac{1}{2} \overline{S^{c}} \mu S+\text { h.c. },
\end{aligned}
$$


where $Y_{D}, Y_{S}, Y_{D S}, M_{R}$ and $\mu$ are all $3 \times 3$ matrices. When the scalar fields $\Phi$ and $\Psi$ get VEVs, the mass matrix for light neutrino sector coming from the effective Lagrangian is given by

$$
\mathcal{M}_{\nu}=\left(\begin{array}{cc}
M_{\nu \nu} & M_{\nu S} \\
M_{\nu S}^{T} & M_{S S}
\end{array}\right)
$$

in the $\left(\nu_{L}^{c}, S\right)^{T}$ basis, where $M_{\nu \nu}, M_{\nu S}$ and $M_{S S}$ are $3 \times 3$ matrices and given respectively by

$$
\begin{aligned}
& M_{\nu \nu}=-m_{D} M_{R}^{-1} m_{D}^{T}, \\
& M_{\nu S}=m_{D S}-m_{D} M_{R}^{-1} m_{S}^{T}, \\
& M_{S S}=\mu-m_{S} M_{R}^{-1} m_{S}^{T},
\end{aligned}
$$

where $m_{D}=Y_{D}\left\langle\phi^{0}\right\rangle, m_{S}=Y_{S}\langle\Psi\rangle$ and $m_{D S}=Y_{D S}\left\langle\phi^{0}\right\rangle$. Note that $M_{\nu \nu}=M_{\nu \nu}^{T}$ and $M_{S S}=M_{S S}^{T}$ are the symmetric $3 \times 3$ Majorana left- and right-handed neutrino mass matrices, respectively. Here we take $M_{R} \gg m_{S} \simeq m_{D} \gg \mu$, and neutrinos become pseudoDirac particles when $M_{\nu S}$ is dominant over $M_{\nu \nu}$ and $M_{S S}$ in eq. (2.3), which reflects $m_{D S} \gg\left(m_{D} m_{S}\right) / M_{R}$. Then, the mass splitting of the light neutrinos depends on the diagonal elements $M_{\nu \nu}$ and $M_{S S}$ in flavor space. And the mixing between active states and sterile ones given as $|\tan 2 \theta|=\left|2 M_{\nu S} /\left(M_{S S}-M_{\nu \nu}\right)\right| \gg 1$ becomes almost maximal.

As shown by Lim and Kobayashi [71], the $6 \times 6$ matrix given in eq. (2.3) can be diagonalized by

$$
W_{\nu}=X \cdot V, \quad \text { with } \quad X=\left(\begin{array}{cc}
U_{L}^{*} & 0 \\
0 & U_{R}
\end{array}\right), \quad V=\left(\begin{array}{cc}
V_{1} & i V_{1} \\
V_{2} & -i V_{2}
\end{array}\right),
$$

where the $3 \times 3$ matrix $U_{L}$ corresponds to Pontecorvo-Maki-Nakagawa-Sakata (PMNS) mixing matrix, the $3 \times 3$ matrix $U_{R}$ is an unknown unitary matrix and $V_{1}$ and $V_{2}$ are the diagonal matrices, $V_{1}=\operatorname{diag}(1,1,1) / \sqrt{2}, V_{2}=\operatorname{diag}\left(e^{-i \phi_{1}}, e^{-i \phi_{2}}, e^{-i \phi_{3}}\right) / \sqrt{2}$ with $\phi_{i}$ being arbitrary phases. The dominant matrix $M_{\nu S}$ in eq. (2.3) can be real and positive diagonalized by biunitary transformation

$$
U_{L}^{\dagger} M_{\nu S} U_{R}=\operatorname{diag}\left(m_{1}, m_{2}, m_{3}\right) \equiv \hat{M} .
$$

Keeping terms up to the first order in heavy Majorana mass, the Hermitian matrix $\mathcal{M}_{\nu}^{\dagger} \mathcal{M}_{\nu}$ can be real and positive diagonalized by a unitary transformation $W_{\nu}$ in eq. (2.5);

$$
W_{\nu}^{\dagger} \mathcal{M}_{\nu}^{\dagger} \mathcal{M}_{\nu} W_{\nu} \equiv \hat{\mathcal{M}}_{\nu}^{2} \simeq\left(\begin{array}{cc}
\hat{M}^{2}+\hat{M}|\delta| & 0 \\
0 & \hat{M}^{2}-\hat{M}|\delta|
\end{array}\right)
$$

where $\delta \equiv \hat{M}_{\nu \nu}^{*}+\hat{M}_{S S}$, and $\hat{\mathcal{M}}_{\nu} \equiv W_{\nu}^{T} \mathcal{M}_{\nu} W_{\nu}=\operatorname{diag}\left(m_{\nu 1}, m_{\nu 2}, m_{\nu 3}, m_{S 1}, m_{S 2}, m_{S 3}\right)$. Here $m_{\nu 2}=\sqrt{m_{\nu 1}^{2}+\Delta m_{\text {Sol }}^{2}}$ and $m_{\nu 3}=\sqrt{m_{\nu 1}^{2}+\Delta m_{\text {Atm }}^{2}}$, with $\Delta m_{\text {Sol }}^{2}$ and $\Delta m_{\text {Atm }}^{2}$, respectively, being the solar and atmospheric mass-squared differences measured in neutrino oscillation experiments. As a result, the three active neutrino states emitted by weak interactions are described in terms of the mass eigenstates as

$$
\nu_{\ell}=U_{\ell k} \frac{1}{\sqrt{2}}\left(\nu_{k}-i S_{k}^{c}\right)
$$


where $\ell$ and $k$ denote flavor and mass eigenstates, respectively, and $U \equiv U_{L}$ is the $3 \times 3$ leptonic PMNS mixing matrix. The diagonal matrix $\delta$ responsible for splitting the Dirac neutrino masses is given by

$$
\delta=\hat{\mu}-\frac{\left(\hat{m}_{D}\right)^{2}}{\hat{M}_{R}}-\frac{\left(\hat{m}_{S}\right)^{2}}{\hat{M}_{R}},
$$

in which the hat stands for a diagonalized mass matrix: $\hat{m}_{D}=U_{L}^{\dagger} m_{D} U_{R}, \hat{m}_{S}=U_{R}^{T} m_{S} U_{R}$, $\hat{\mu}=U_{R}^{T} \mu U_{R}$, and $\hat{M}_{R}^{-1}=U_{R}^{\dagger} M_{R}^{-1} U_{R}^{*}$. It is easy to see that the size of $\delta$ is very small compared with the magnitude of $m_{D(S)}$ in the case that the lepton number violating parameter $\mu$ is small and $m_{D(S)} \ll M_{R}$. Then the mass squared difference between $m_{\nu_{i}}$ and $m_{S_{i}}, \Delta m_{k}^{2}\left(=2 m_{k}\left|\delta_{k}\right|\right)$, can be small and thus the pairs of the active and sterile neutrinos can form pseudo-Dirac pairs. It is anticipated that $\Delta m_{k}^{2} \ll \Delta m_{\text {Sol }}^{2},\left|\Delta m_{\text {Atm }}^{2}\right|$, otherwise the effects of the pseudo-Dirac neutrinos should have been detected. But, in the limit that $\Delta m_{k}^{2}=0$, it is hard to discern the pseudo-Dirac nature of neutrinos. The largest $m_{k}^{2}$ value depends on the neutrino mass hierarchy: for normal neutrino mass hierarchy (NH), $m_{3}^{2} \gtrsim \Delta m_{\text {Atm }}^{2} \simeq 2.5 \times 10^{-3} \mathrm{eV}^{2}$ and $m_{2}^{2} \gtrsim \Delta m_{\text {Sol }}^{2} \simeq 7.5 \times 10^{-5} \mathrm{eV}^{2}$, while for inverted one (IH) $m_{2}^{2}>m_{1}^{2} \gtrsim 2.5 \times 10^{-3} \mathrm{eV}^{2}$. Thus, the upper bounds for the values of $\delta_{k}$ are given by

$$
\left|\delta_{1}\right| \ll 3.8 \times 10^{-5} \mathrm{eV}^{2} / m_{1}, \quad\left|\delta_{2}\right| \ll 4.3 \times 10^{-3} \mathrm{eV}, \quad\left|\delta_{3}\right| \ll 7.5 \times 10^{-4} \mathrm{eV},
$$

for $\mathrm{NH}$, and for $\mathrm{IH}$

$$
\left|\delta_{1,2}\right| \ll 7.5 \times 10^{-4} \mathrm{eV}, \quad\left|\delta_{3}\right| \ll 3.8 \times 10^{-5} \mathrm{eV}^{2} / m_{3} .
$$

Note that those values are very crucial for a successful low scale leptogenesis as will be discussed later. In case of $|\hat{\mu}| \ll\left|\hat{m}_{D}^{2} / \hat{M}_{R}\right|$, the very tiny mass splitting between active and sterile neutrinos arises from lepton number violating dimension-5 operators suppressed by a very high energy scale (e.g. the GUT scale or Planck scale): for example, assuming normal mass hierarchy and taking $M_{R} \sim$ Planck mass $\sim 1.22 \times 10^{19} \mathrm{GeV}$, then the tiny mass splittings are $\Delta m_{1}^{2} \ll 8.6 \times 10^{-8} y_{1}^{2} \mathrm{eV}^{2}, \Delta m_{2}^{2} \simeq 8.6 \times 10^{-8} y_{2}^{2} \mathrm{eV}^{2}$, and $\Delta m_{3}^{2} \simeq$ $5 \times 10^{-7} y_{3}^{2} \mathrm{eV}^{2}$, where $y_{i}$ are the diagonal entries of $\hat{Y}_{D}$ or $\hat{Y}_{S}$. On the other hand, for $|\hat{\mu}| \gg\left|\hat{m}_{D}^{2} / \hat{M}_{R}\right|$, the tiny mass splittings are governed by $\delta_{k} \simeq\left|\hat{\mu}_{k}\right|$. Interestingly enough, the upper bound for $\left|\delta_{1}\right|\left(\left|\delta_{3}\right|\right)$ could be large enough according to the lightest neutrino mass $m_{\nu_{1}} \approx m_{1}\left(m_{\nu_{3}} \approx m_{3}\right)$ for $\mathrm{NH}(\mathrm{IH})$. As will be seen in eq. (3.12), a successful TeV-scale leptogenesis could be viable even for a hierarchical heavy neutrino spectrum in such a way that, as the lightest neutrino mass gets lower, the corresponding scale of $\delta_{1}$ or $\delta_{3}$ increases.

\section{Leptogenesis with pseudo-Dirac neutrinos}

Now, let us consider how low scale leptogenesis ${ }^{1}$ can be successfully generated in this scenario by decay of the lightest right-handed Majorana neutrino before the scalar fields get vacuum expectation values. In particular, there is a new contribution to the lepton asymmetry which is mediated by the extra singlet neutrinos.

\footnotetext{
${ }^{1}$ See also leptogenesis in inverse seesaw neutrino models [72, 73].
} 

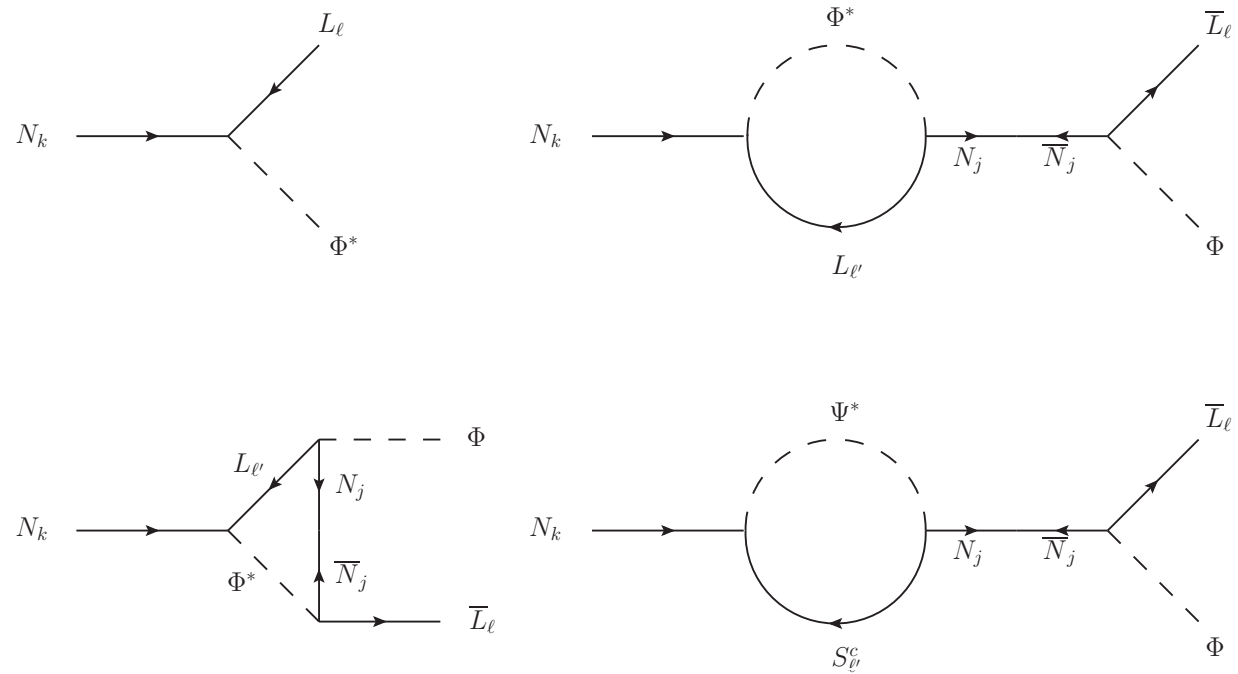

Figure 1. Diagrams contributing to lepton asymmetry.

Without loss of generality, we can rotate and rephase the fields to make the mass matrices $M_{R_{i j}}$ and $\mu_{i j}$ real and diagonal. In this basis, the elements of $Y_{D}$ and $Y_{S}$ are in general complex. As shown in figure 1, the lepton number asymmetry from decay of the right-handed heavy neutrino into leptons and Higgs scalar required for baryogenesis is given by

$$
\varepsilon_{k}=\sum_{\ell}\left[\frac{\Gamma\left(N_{k} \rightarrow L_{\ell} \Phi^{*}\right)-\Gamma\left(N_{k} \rightarrow \bar{L}_{\ell} \Phi\right)}{\Gamma_{\mathrm{tot}}\left(N_{k}\right)}\right]
$$

where $N_{k}$ is the decaying right-handed neutrino and $\Gamma_{\text {tot }}\left(N_{k}\right)$ is the total decay rate. In addition to the diagrams of the standard leptogenesis scenario, there is a new contribution of the diagram which corresponds to the self energy correction of the vertex arisen due to the new Yukawa couplings with singlet neutrinos and Higgs sectors. Assuming that the masses of the Higgs sectors and extra singlet neutrinos are much smaller compared to that of the right-handed neutrino, to leading order, we have

$$
\Gamma_{\text {tot }}\left(N_{k}\right)=\frac{\left(Y_{D}^{\dagger} Y_{D}+Y_{S}^{\dagger} Y_{S}\right)_{k k}}{4 \pi} M_{R_{k}}
$$

so that

$$
\varepsilon_{k}=\frac{1}{8 \pi} \sum_{j \neq k}\left(\left[g_{V}\left(x_{j}\right)+g_{S}\left(x_{j}\right)\right] \mathcal{T}_{k j}+g_{S}\left(x_{j}\right) \mathcal{S}_{k j}\right),
$$

where $g_{V}\left(x_{j}\right)=\sqrt{x_{j}}\left\{1-\left(1+x_{j}\right) \ln \left[\left(1+x_{j}\right) / x_{j}\right]\right\}, g_{S}\left(x_{j}\right)=\sqrt{x_{j}} /\left(1-x_{j}\right)$ with $x_{j}=M_{R_{j}}^{2} / M_{R_{k}}^{2}$ for $j \neq k$,

$$
\mathcal{T}_{k j}=\frac{\operatorname{Im}\left[\left(Y_{D}^{\dagger} Y_{D}\right)_{k j}^{2}\right]}{\left(Y_{D}^{\dagger} Y_{D}+Y_{S}^{\dagger} Y_{S}\right)_{k k}}, \quad \mathcal{S}_{k j}=\frac{\operatorname{Im}\left[\left(Y_{D}^{\dagger} Y_{D}\right)_{k j}\left(Y_{S}^{\dagger} Y_{S}\right)_{k j}\right]}{\left(Y_{D}^{\dagger} Y_{D}+Y_{S}^{\dagger} Y_{S}\right)_{k k}} .
$$

Notice that the term proportional to $\mathcal{S}_{k j}$ comes from the interference of the tree-level diagram with new contribution mediated by $S$. 
The newly generated $B-L$ asymmetry is given as $Y_{B-L}^{\mathrm{SM}}=-\eta \varepsilon_{1} Y_{N_{1}}^{\mathrm{eq}}$, where $Y_{N_{1}}^{\mathrm{eq}}$ is the number density of the right-handed heavy neutrino at $T \gg M_{R_{1}}$ in thermal equilibrium given as $Y_{N_{1}}^{\text {eq }} \simeq \frac{45}{\pi^{4}} \frac{\zeta(3)}{g_{*} k_{B}} \frac{3}{4}$ with Boltzmann constant $k_{B}$ and the effective number of degree of freedom $g_{*}\left(g_{*}=217 / 2\right.$ for the SM) [74]. The efficient factor $\eta$ can be computed through a set of coupled Boltzmann equations which take into account processes that create or washout the asymmetry. For successful leptogenesis, the size of the denominator of $\varepsilon_{1}$ should be constrained by the out-of-equilibrium condition, $\Gamma_{N_{1}}<\left.H\right|_{T=M_{R_{1}}}$, where $\Gamma_{N_{1}}$ is the total decay width of $N_{1}$ and $H\left(T=M_{R_{1}}\right)=\sqrt{\frac{4 \pi^{3} g_{*}}{45}} \frac{M_{R_{1}}^{2}}{M_{\mathrm{Pl}}}$ is the Hubble parameter at temperature $T=M_{R_{1}}$. The efficiency in generating the resultant baryon asymmetry is usually controlled by the parameter defined as

$$
K \equiv \frac{\sum_{\ell} \Gamma\left(N_{1} \rightarrow L_{\ell} \Phi^{*}\right)}{H\left(T=M_{R_{1}}\right)}=\frac{\tilde{m}_{1}}{m_{*}} .
$$

We note that $K \ll 1$ corresponds to weak washout, whereas $K \gg 1$ to strong washout. To a good approximation the efficiency factor depends on the effective neutrino mass $\tilde{m}_{1}$ defined in the presence of the new Yukawa interactions with the coupling $Y_{S}$ by

$$
\tilde{m}_{1}=\frac{\left(Y_{D}^{\dagger} Y_{D}+Y_{S}^{\dagger} Y_{S}\right) 11}{M_{R_{1}}} v^{2} \sim 2 \delta_{1},
$$

which is a measure of the strength of the coupling of $N_{1}$ to the thermal bath. And the equilibrium neutrino mass is given by $m_{*}=\frac{16 \pi^{5 / 2}}{3 \sqrt{5}} \sqrt{g_{*}} \frac{v^{2}}{M_{\mathrm{Pl}}} \simeq 1.08 \times 10^{-3} \mathrm{eV}$. Note here that the plausible range for $\tilde{m}_{1}$ is the one suggested by the range of the order of the $\delta_{1}$. In such a case, the decay rate is smaller than the expansion rate of the universe, and the particles come out of equilibrium and create a lepton asymmetry. So, the produced baryon asymmetry depends on the initial conditions in the weak washout regime. The efficiency factor for $0<\eta<1$ can be estimated by inserting this effective mass in the function [75]

$$
\eta(x) \simeq\left(\frac{3.3 \times 10^{-3} \mathrm{eV}}{x}+\left(\frac{x}{5.5 \times 10^{-4} \mathrm{eV}}\right)^{1.16}\right)^{-1}
$$

valid for $M_{R_{1}} \ll 10^{14} \mathrm{GeV}$. Then, the baryon-to-photon ratio results in $\eta_{B} \simeq-0.97 \times$ $10^{-2} \times \eta\left(\tilde{m}_{1}\right) \times \varepsilon_{1}$. From the observed one in nine year WMAP data $[76,77] \eta_{B}^{\text {WMAP }}=$ $(6.19 \pm 0.14) \times 10^{-10}$, we can get the allowed range of the model parameter $\delta_{i}$ and some bounds on $M_{R_{1}}$,as will be shown later.

In a hierarchical pattern for right-handed neutrinos $M_{2,3} \gg M_{1}$, it is sufficient to consider the lepton asymmetry produced by the decay of the lightest right-handed neutrino $N_{R_{1}}$ :

$$
\varepsilon_{1} \simeq \frac{3}{16 \pi} \frac{M_{R_{1}}}{\left(m_{D}^{\dagger} m_{D}+m_{S}^{\dagger} m_{S}\right)_{11}} \operatorname{Im}\left[\left(Y_{D}^{\dagger} M_{\nu \nu} Y_{D}^{*}\right)_{11}\right],
$$

where $\langle\Psi\rangle \simeq\left\langle\phi^{0}\right\rangle=v$ is used and the loop function $g_{V}$ can be approximated as $g_{V}\left(x_{j}\right)=$ $-\frac{3}{2 \sqrt{x_{j}}}$ for $x_{j} \gg 1$. Using the relation below eq. (2.7), i.e. real and positive eigenvalues $\hat{M}_{\nu \nu}=U_{L}^{\dagger} M_{\nu \nu} U_{L}^{*}$, we have

$$
\operatorname{Im}\left[\left(\tilde{Y}_{D}^{\dagger} \hat{M}_{\nu \nu} \tilde{Y}_{D}^{*}\right)_{11}\right]=\sum_{j=1}^{3} \operatorname{Im}\left[\left(\tilde{Y}_{D}^{\dagger}\right)_{1 j}^{2}\right]\left(\hat{M}_{\nu \nu}\right)_{j} \simeq \frac{1}{2} \sum_{j=1}^{3} \operatorname{Im}\left[\left(\tilde{Y}_{D}^{\dagger}\right)_{1 j}^{2}\right]\left|\delta_{j}\right|,
$$


where $\tilde{Y}_{D}=U_{L}^{\dagger} Y_{D}$, and the third equality comes out from $|\hat{\mu}| \ll\left|\hat{m}_{D}^{2} / M_{R}\right|$ with $\hat{m}_{D} \sim \hat{m}_{S}$. By letting the three-vector $\hat{Y}_{1 j}^{\dagger}=\left(\tilde{Y}_{D}^{\dagger}\right)_{1 j} / \sqrt{\left(\tilde{Y}_{D}^{\dagger} \tilde{Y}_{D}\right)_{11}}$, and simply taking $\left(m_{D}^{\dagger} m_{D}\right)_{11} \simeq$ $\left(m_{S}^{\dagger} m_{S}\right)_{11}$, the lepton asymmetry $\varepsilon_{1}$ in eq. (3.8) can be simplified as

$$
\varepsilon_{1} \simeq \frac{3 M_{R_{1}}}{64 v^{2} \pi} \sum_{j=1}^{3} \operatorname{Im}\left[\left(\hat{Y}_{1 j}^{\dagger}\right)^{2}\right]\left|\delta_{j}\right| \leq \frac{3 M_{R_{1}}}{64 v^{2} \pi} \delta_{\max }=\varepsilon_{1}^{\max },
$$

where $\delta_{\max }$ is the heaviest $\left|\delta_{j}\right|$. Interestingly enough, we estimate what values of $\delta_{\max }$ can be obtained from the solar neutrino data and GRB neutrinos

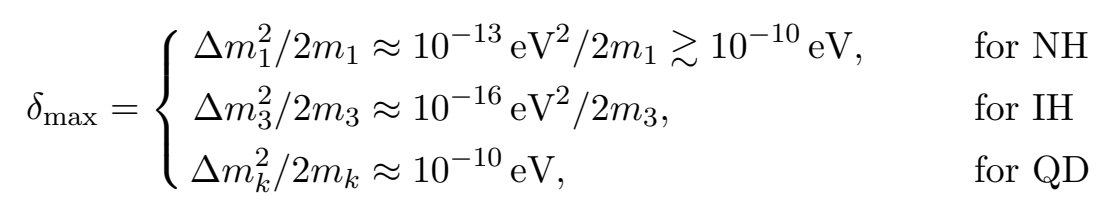

in which $\Delta m_{1}^{2} \sim 10^{-13} \mathrm{eV}^{2}$ and $\Delta m_{3}^{2} \sim 10^{-16} \mathrm{eV}^{2}$ are taken, the QD stands for quasidegenerate neutrino mass, and the lower bound for NH is achieved when both $m_{1}$ and $m_{2}$ are of the same order. The maximal CP asymmetry $\varepsilon_{1}^{\max }$ then yields the maximal baryon asymmetry $\eta_{B}^{\max }$ that can be produced in leptogenesis. The lower bound on $\left|\varepsilon_{1}\right|$ and the upper bound on $\delta_{\max }$ can be used to obtain a lower bound on $M_{R_{1}}$

$$
M_{R_{1}} \gtrsim 1 \mathrm{TeV}\left(\frac{0.2 \mathrm{MeV}}{\delta_{\max }}\right)\left(\frac{\left|\varepsilon_{1}\right|}{10^{-7}}\right)
$$

which means for $\delta_{\max } \sim 0.2 \mathrm{MeV}$, the lower bound on the scale of lightest heavy neutrino will be of the order of $\gtrsim 1 \mathrm{TeV}$ for a successful leptogenesis. Here the $\delta_{\max } \sim 0.2 \mathrm{MeV}$ in the normal neutrino mass hierarchy can be obtained for $\hat{m}_{D_{1}} \sim 0.3 \mathrm{GeV}$ and $\left|M_{R_{1}}\right| \sim 1 \mathrm{TeV}$ in the limit of $\left|\hat{\mu}_{1}\right| \ll\left|\left(\hat{m}_{D_{1}}\right)^{2} / M_{R_{1}}\right|$. One can obtain the $\delta_{\max }$ for IH in similar way. On the other hand, in the case of QD, i.e. $m_{\nu_{1}} \approx m_{\nu_{2}} \approx m_{\nu_{3}}$, the lower bound on the scale of lightest heavy neutrino will be located around $M_{R} \sim$ Planck scale for a successful leptogenesis. In its simplest scenario, thermal leptogenesis, since the baryon asymmetry is produced during the radiation dominated era, the lower bound on $M_{R_{1}} \gtrsim 1 \mathrm{TeV}$ with $\delta_{\max } \sim 0.2 \mathrm{MeV}$ for a hierarchical neutrino mass translates into a lower bound on the reheating temperature after inflation.

On the other hand, in a case $\delta_{\max } \sim 2 \times 10^{-4} \mathrm{eV}$, the lower bounds on the scale of lightest heavy neutrino will be of the order of $M_{R} \gtrsim 10^{12} \mathrm{GeV}$ for a successful leptogenesis, which translates into a lower bound on the reheating temperature after inflation. ${ }^{2}$ In such a case, the lower bound on the reheating temperature can be relaxed by considering quasi-degenerate heavy Majorana neutrinos $\left(M_{R_{1}} \simeq M_{R_{2}}\right)$ [78]. So in its form, thermal production of $N_{1}$ does not need too high reheating temperature and the Universe would not encounter the gravitino overproduction $[79,80]$. In the following we will see this is the

\footnotetext{
${ }^{2}$ Such a large reheating temperature is potentially in conflict with bigbang nucleosynthesis (BBN) in supersymmetric models, where upper bound on the reheating temperature as low as $10^{6} \mathrm{GeV}$ unless $m_{3 / 2} \gg$ $1 \mathrm{TeV}$ have been obtained in supergravity (SUGRA) models [79].
} 
case. As shown in [81-84], the new contributions to $\varepsilon_{1}$ could be important for the case of $M_{R_{1}} \simeq M_{R_{2}}<M_{R_{3}}$ for which the asymmetry is approximately given by

$$
\varepsilon_{1} \simeq \frac{M_{R_{2}}}{16 \pi} \frac{\operatorname{Im}\left[\left(Y_{D}^{\dagger} M_{\nu \nu} Y_{D}^{*}\right)_{11}\right]-\operatorname{Im}\left[\left(Y_{D}^{\dagger}\left(m_{D} M_{R}^{-1} m_{S}^{T}\right) Y_{S}^{*}\right)_{11}\right]}{\left(m_{D}^{\dagger} m_{D}+m_{S}^{\dagger} m_{S}\right)_{11}} R
$$

where $R$ is a resonance factor defined by $R \equiv\left|M_{R_{1}}\right| /\left(\left|M_{R_{2}}\right|-\left|M_{R_{1}}\right|\right)$. In the above equation the denominator can be expressed as

$$
\begin{gathered}
\sum_{j}\left(\operatorname{Im}\left[\left(\tilde{Y}_{D}^{\dagger}\right)_{1 j}^{2}\right]\left(\hat{M}_{\nu \nu}\right)_{j}+\operatorname{Im}\left[\left(\tilde{Y}_{D}^{\dagger}\right)_{1 j}\left(\tilde{Y}_{S}^{\dagger}\right)_{1 j}\right]\left\{\hat{M}_{j}-\left(\hat{m}_{D S}\right)_{j}\right\}\right) \\
\simeq \sum_{j}\left\{\operatorname{Im}\left[\left(\tilde{Y}_{D}^{\dagger}\right)_{1 j}^{2}+\left(\tilde{Y}_{D}^{\dagger}\right)_{1 j}\left(\tilde{Y}_{S}^{\dagger}\right)_{1 j}\right]\right\} \frac{\left|\delta_{j}\right|}{2}
\end{gathered}
$$

where $\tilde{Y}_{S}=U_{R}^{T} Y_{S}$, and the second equality comes out from $|\hat{\mu}| \ll\left|\hat{m}_{D}^{2} / M_{R}\right|$ with $\hat{m}_{D} \sim \hat{m}_{S}$ leading to $\hat{M}_{j}-\left(\hat{m}_{D S}\right)_{j} \simeq\left|\delta_{j}\right| / 2$ as well as $\left(\hat{M}_{\nu \nu}\right)_{j} \simeq\left|\delta_{j}\right| / 2$. So, we obtain

$$
\varepsilon_{1} \simeq \frac{M_{R_{2}}}{32 v^{2} \pi} \frac{\sum_{j}\left\{\operatorname{Im}\left[\left(\tilde{Y}_{D}^{\dagger}\right)_{1 j}^{2}+\left(\tilde{Y}_{D}^{\dagger}\right)_{1 j}\left(\tilde{Y}_{S}^{\dagger}\right)_{1 j}\right]\right\}\left|\delta_{j}\right|}{\left(\tilde{Y}_{D}^{\dagger} \tilde{Y}_{D}+\tilde{Y}_{S}^{\dagger} \tilde{Y}_{S}\right)_{11}} R \leq \frac{M_{R_{2}}}{64 v^{2} \pi} \delta_{\max } R .
$$

Similar to the hierarchical case, the lower bound on $M_{R_{2}}$ can be obtained by using both the upper bound on $\left|\varepsilon_{1}\right|$ and the lower bound on $\delta_{\max }$,

$$
M_{R_{2}} \gtrsim 3 \times 10^{12-n} \mathrm{GeV}\left(\frac{2 \times 10^{-4} \mathrm{eV}}{\delta_{\max }}\right)\left(\frac{10^{n}}{R}\right)\left(\frac{\left|\varepsilon_{1}\right|}{10^{-7}}\right) .
$$

This lower bound on $M_{R_{2}}$ further implies a lower bound of the reheating temperature after inflation, since the abundance of gravitinos is proportional to the reheating temperature. The degree of degeneracy between two heavy neutrinos $R=10^{6-9}$ is required to achieve a successful leptogenesis, corresponding to the lower bound on $M_{R_{2}} \gtrsim 3 \times 10^{3-6} \mathrm{GeV}$ for $\delta_{\max } \sim 2 \times 10^{-4} \mathrm{eV}$.

\section{Probing pseudo-Dirac neutrinos at astronomical-scale experiments}

Now, let us consider how one can probe the effects of the pseudo-Dirac neutrinos. A possible way to probe the pseudo-Dirac neutrinos is to perform astronomical-scale baseline experiments to uncover the oscillation effects of very tiny mass splitting $\Delta m_{k}^{2}$. With the help of the mixing matrix eq. (2.5), the flavor conversion probability between the active neutrinos follows from the time evolution of the state $\nu_{k}$ as,

$$
P_{\ell \ell^{\prime}} \equiv P_{\nu_{\ell} \rightarrow \nu_{\ell^{\prime}}}\left(W_{\nu}, L, E\right)=\left|\left(W_{\nu}^{*} e^{-i \frac{\hat{\mathcal{M}}_{\nu}^{2}}{2 E} L} W_{\nu}^{T}\right)_{\ell \ell^{\prime}}\right|^{2}=\frac{1}{4}\left|\sum_{k=1}^{3} U_{\ell^{\prime} k}\left\{e^{i \frac{m_{\nu k}^{2} L}{2 E}}+e^{i \frac{m_{S k}^{2} L}{2 E}}\right\} U_{\ell k}^{*}\right|^{2},
$$

where $W_{\nu}$ is the mixing matrix with which the weak gauge eigenstates, $\nu_{\ell}$, with flavor $\ell=e, \mu, \tau$ are composed of the mass eigenstates with definite masses, $n_{k}=\left(\nu_{k} S_{k}^{c}\right)^{T}(k=$ $1,2,3)$, giiven as $\left|\nu_{\ell}\right\rangle=\sum_{k}^{N_{\nu}=3} W_{\ell k}^{*}\left|n_{k}\right\rangle$. 
Neutrinos arriving at neutrino telescopes from astrophysical sources such as Gamma Ray Bursts (GRBs) [85, 86], active galactic nuclei [87-89], and type Ib/c supernova [9093] travel large distances over $\sim 100 \mathrm{Mpc}$. Neutrino telescope observes neutrinos from extragalactic sources located a few Gpc away from the earth and with neutrino energy $10^{5} \mathrm{GeV} \lesssim E \lesssim 10^{7} \mathrm{GeV}$. It has been shown [94] that inside the GRB sources $\int V_{C, N} d t \ll 1$ where the effective potentials due to the matter effects are $V_{C}=\sqrt{2} G_{F} n_{e}$ with $n_{e}$ being the electron number density in matter and $V_{N}=-\sqrt{2} G_{F} n_{n} / 2$ with $n_{n}$ being the neutron number density in matter, so the matter effects inside the source are not relevant for neutrino oscillation, while inside the earth for $V_{C, N} \gg \Delta m_{k}^{2} / 2 E$ again the matter effect will not be significant because of the very tiny effective mixing angle. So, we only consider neutrino oscillation in vacuum for astrophysical neutrinos. Given neutrino trajectory $L$ and energy $E$, the oscillation effects become prominent when $\Delta m_{k}^{2} \sim E / 4 \pi L$, where $L \equiv L(z)$ is a distance-measure with redshift $z$ given by $[46,47]$

$$
L(z) \equiv D_{H} \int_{0}^{z} \frac{d z^{\prime}}{\left(1+z^{\prime}\right)^{2} \sqrt{\Omega_{m}\left(1+z^{\prime}\right)^{3}+\Omega_{\Lambda}}},
$$

where the Hubble length $D_{H}=c / H_{0} \simeq 4.42 \mathrm{Gpc}$ with the present Hubble expansion rate $H_{0}=67.8 \pm 0.9 \mathrm{~km} \mathrm{~s}^{-1} \mathrm{Mpc}^{-1}$ [48], the matter density of the Universe $\Omega_{m}=0.306 \pm 0.007$, and the dark energy density of the Universe $\Omega_{\Lambda}=0.694 \pm 0.007$ [49]. The asymptotic value of $L(z)$ is about $2.1 \mathrm{Gpc}$ achieved by large value of $z$, which means that the smallest $\Delta m_{k}^{2}$ that can be probed with astrophysical neutrinos with $E$ is $10^{-17} \mathrm{eV}^{2}(E / \mathrm{PeV})[46,47]$. Thus, astrophysical neutrinos with $L \simeq 1 \mathrm{Gpc}$ (the flight length) and energy $E \simeq 1 \mathrm{PeV}$ would be useful to probe the pseudo-Dirac property of neutrinos with very tiny mass splitting. In this case, to observe the oscillation effects, the oscillation lengths should not be much larger than the flight length before arriving at neutrino telescopes in earth, that is,

$$
L_{\mathrm{osc}}^{k} \simeq\left(\frac{0.8 \times 10^{-16} \mathrm{eV}^{2}}{\Delta m_{k}^{2}}\right)\left(\frac{E}{10^{6} \mathrm{GeV}}\right) \mathrm{Gpc} \lesssim \mathrm{Gpc}
$$

which means that neutrino oscillations can be measurable only when $\delta_{k} \gtrsim 0.8 \times 10^{-15} \mathrm{eV}$. From eq. (4.3), we see that given the tiny mass splittings $\Delta m_{k}^{2}=10^{-16 \sim-17} \mathrm{eV}^{2}$ with the energies around $\mathrm{TeV}-\mathrm{PeV}$, a new oscillation curve at neutrino trajectory $\mathcal{O}(1) \mathrm{Gpc}$ is naively expected to occur. Since $E / \Delta m_{k}^{2} \sim L(z) \gg E / \Delta m_{\text {Atm }}^{2}$, the probability of the oscillation $\nu_{\mu} \rightarrow \nu_{\mu}$ over the distance $L$ is given approximately by

$$
\begin{aligned}
P\left(\nu_{\mu} \rightarrow \nu_{\mu}\right) \simeq 1 & -\frac{1}{2} c_{23}^{4}\left\{4 s_{23}^{2}+\sin ^{2} 2 \theta_{12} c_{23}^{2}+\sin 2 \theta_{23} \sin 4 \theta_{12} \cos \delta_{\mathrm{CP}} s_{13}\right\} \\
& -s_{23}^{4} \sin ^{2}\left(\frac{\Delta m_{3}^{2} L}{4 E}\right)-c_{23}^{4}\left\{s_{12}^{4} \sin ^{2}\left(\frac{\Delta m_{2}^{2} L}{4 E}\right)+c_{12}^{4} \sin ^{2}\left(\frac{\Delta m_{1}^{2} L}{4 E}\right)\right\} \\
& -\sin 2 \theta_{23} \sin 2 \theta_{12} \cos \delta_{\mathrm{CP}} s_{13} c_{23}^{2}\left\{c_{12}^{2} \sin ^{2}\left(\frac{\Delta m_{2}^{2} L}{4 E}\right)-s_{12}^{2} \sin ^{2}\left(\frac{\Delta m_{1}^{2} L}{4 E}\right)\right\}
\end{aligned}
$$

where we have ignored the terms proportional to $\sin ^{n} \theta_{13}$ with $n \geq 2$. For the numerical analysis, we take the results from global fit of three-flavor oscillation parameters at $1 \sigma$ C.L. [95], which are given in table 1. 


\begin{tabular}{|c|c|c|c|c|}
\hline mass hierarchy & $\theta_{23}\left(^{\circ}\right)$ & $\theta_{12}\left(^{\circ}\right)$ & $\theta_{13}\left(^{\circ}\right)$ & $\delta_{\mathrm{CP}}\left(^{\circ}\right)$ \\
\hline normal & $42.3_{-1.6}^{+3.0}$ & $33.48_{-0.75}^{+0.78}$ & $8.50_{-0.21}^{+0.20}$ & $306_{-70}^{+39}$ \\
\hline inverted & $49.5_{-2.2}^{+1.5}$ & $33.48_{-0.75}^{+0.78}$ & $8.51_{-0.21}^{+0.20}$ & $254_{-62}^{+63}$ \\
\hline
\end{tabular}

Table 1. Global fit of three-flavor neutrino oscillation parameters at $1 \sigma$.

Recently, authors in ref. [70] analyzed the high energy neutrino events observed by IceCube, aiming to probe the initial flavor of cosmic neutrinos. The expected number of events produced by an isotropic neutrino and antineutrino with flavor $\ell$ is given by

$$
N=4 \pi T \int d E \Phi_{\ell}(E) A_{\ell}(E)
$$

where $T$ is the time of observation, $A_{\ell}(E)$ is the detector effective areas, and $\Phi_{\ell}(E)$ is the energy dependent isotropic flux of neutrinos and antineutrinos [70]. Then the track-toshower ratio for the number of shower $N_{S}$ and track events $N_{T}$ in the IceCube detector ${ }^{3}$ can be expressed in terms of tiny mass splittings $\Delta m_{k}^{2}$, flight length $L$, neutrino mixing angles and CP phase $\left(\theta_{12}, \theta_{23}, \theta_{13}, \delta_{\mathrm{CP}}\right)$, and initial flavor composition $\phi_{\ell^{\prime}}^{0}$

$$
\frac{N_{T}}{N_{S}}=\frac{a_{\mu} p_{T} \tilde{F}_{\mu}}{a_{e} \tilde{F}_{e}+a_{\mu}\left(1-p_{T}\right) \tilde{F}_{\mu}+a_{\tau} \tilde{F}_{\tau}},
$$

where

$$
\begin{aligned}
& \tilde{F}_{\ell}=\sum_{\ell^{\prime} k}\left|U_{\ell k}\right|^{2}\left|U_{\ell^{\prime} k}\right|^{2} \phi_{\ell^{\prime}}^{0} \\
& a_{\ell}=4 \pi \int d E \cos ^{2}\left(\frac{\Delta m_{k}^{2} L}{4 E}\right) E^{-\alpha} A_{\ell}(E),
\end{aligned}
$$

with a spectral index $\alpha$. Here $p_{T}$ is the probability that an observed event produced by a muon neutrino is a track event, which is mildly dependent on energy and approximately equals to 0.8 [96]. Then above equation can be simplified to

$$
\frac{N_{T}}{N_{S}}=\frac{\phi_{\mu}}{\frac{a_{e}}{a_{\mu} p_{T}}+\left(\frac{a_{\tau}}{a_{\mu} p_{T}}-\frac{a_{e}}{a_{\mu} p_{T}}\right) \phi_{\tau}+\left(\frac{1-p_{T}}{p_{T}}-\frac{a_{e}}{a_{\mu} p_{T}}\right) \phi_{\mu}},
$$

where $\phi_{e}=1-\phi_{\mu}-\phi_{\tau}$ with $\phi_{\ell} \equiv \tilde{F}_{\ell} /\left(\tilde{F}_{e}+\tilde{F}_{\mu}+\tilde{F}_{\tau}\right)$ is assumed. By using the high energy neutrino events in the IceCube detector which lie in energies between $60 \mathrm{TeV}$ and $3 \mathrm{PeV}[68-$ 70], eq. (4.8) shows directly that track-to-shower ratio $N_{T} / N_{S}$ can give a new oscillation curve as a signal dependent on neutrino flight length if the neutrino mixing angles and $\mathrm{CP}$ phase, initial flavor composition, and tiny mass splittings are given as inputs.

In the limit of large or null mass splitting $\Delta m_{k}^{2}$, there is no oscillation effects and thus the value of $N_{T} / N_{S}$ becomes constant for a given data set of neutrino mixing angles

\footnotetext{
${ }^{3}$ We note that much larger detectors than the present IceCube would be required to get fully meaningful result for the test of our model in detail.
} 
and $\mathrm{CP}$ phase. However, in the case that the oscillation effects are prominent, the value of $N_{T} / N_{S}$ can be enhanced due to the new oscillatory term which depends on neutrino flight length, and small mass splittings. Thus, it is possible to probe the pseudo-Dirac property of neutrinos by measuring the deviation of $N_{T} / N_{S}$ from the expectation without the oscillation arisen due to the tiny mass splitting. To see how large the value of $N_{T} / N_{S}$ can be deviated by the oscillatory terms, we perform numerical analysis by taking the values of the neutrino mixing angles and $\mathrm{CP}$ phase from the global fit results at $1 \sigma$ level [95] as shown in table 1 . We expect that the different values of $\theta_{23}$ and $\delta_{\mathrm{CP}}$ at $1 \sigma$ level for normal and inverted mass orderings provide different predictions of the track-to-shower ratio, while normal and inverted mass orderings could not be distinguished with the data at $3 \sigma$ level [95]. For the tiny mass splittings, we consider two cases: (i) all equivalent, $\Delta m_{1,2,3}^{2}=\Delta m_{k}^{2}$, and (ii) hierarchical $\Delta m_{i}^{2} \gg \Delta m_{j}^{2}$ for $(i>j)$.

Our numerical results depend on the initial flavor composition $\phi_{e}^{0}: \phi_{\mu}^{0}: \phi_{\tau}^{0}$ at the source which are relevant for the interpretation of observational data. We consider the well-known four production mechanisms for high energy neutrinos from which the flavor compositions are given as: (i) $\left(\frac{1}{3}: \frac{2}{3}: 0\right)$ for $\pi$ decay, (ii) $\left(\frac{1}{2}: \frac{1}{2}: 0\right)$ for charmed mesons decay, (iii) $(1: 0: 0)$ for $\beta$ decay of neutrons, and (iv) $(0: 1: 0)$ for $\pi$ decay with damped muons. The tiny mass splittings $\Delta m_{k}^{2}$ can be searched for, looking at high energy cosmic neutrinos by measuring the track-to-shower ratio $N_{T} / N_{S}$ as the function of $L\left(\log _{10}\right.$ [path length/Mpc]) in eq. (4.8). In the numerical analysis, we use the spectral index given by $\alpha=2.2 \pm 0.4$ [96].

\subsection{Results for the case of $\Delta m_{1,2,3}^{2}=\Delta m_{k}^{2}$}

As a benchmark point, we take the mass splittings $\Delta m_{1,2,3}^{2}$ to be $10^{-16} \mathrm{eV}^{2}$. In figure 2, we present the track-to-shower ratio $N_{T} / N_{S}$ for normal (inverted) mass ordering as a function of $L\left(\log _{10}\right.$ [path length/Mpc]). The red (dark black) and blue (light black) curves correspond to normal and inverted neutrino mass orderings, respectively, for $\alpha=2.2$. The light red and light blue regions correspond to normal and inverted neutrino mass orderings, respectively, for $1.8 \lesssim \alpha \lesssim 2.6$. The gray shaded regions are forbidden by the measurement of $N_{T} / N_{S}=0.18_{-0.05}^{+0.13}$ which is obtained in ref. [70]. In each panel, we present the initial flavor composition for neutrino flux. The width of each band in the panels represent the uncertainties in the measurements of neutrino mixing angles. From figure 2, we see that the bands for the cases with only one flavor in the initial flavor composition are wider than the others. This is because of the slightly hierarchical neutrino mixing angles and the initial flavor compositions with $\phi_{\ell}$ in eq. (4.8): for example, the main reason in the different band widths of the left upper and lower panels in figure 2 is the initial flavor compositions with $\phi_{\ell}$, while the main reason in the different band widths of the left-lower and right-lower panels is the slightly hierarchical neutrino mixing angles. The results in the lower panels show that the predicted values of $N_{T} / N_{S}$ for normal mass ordering are consistent with the boundaries of the allowed region of $N_{T} / N_{S}$. In the upper panels, we see that the oscillation peak occurs at the distance about $1.3 \mathrm{Gpc}$ for both the tiny mass splittings $\Delta m_{1,2,3}^{2}=10^{-16} \mathrm{eV}^{2}$ and the deposited energies $60 \mathrm{TeV}-3 \mathrm{PeV}$. 

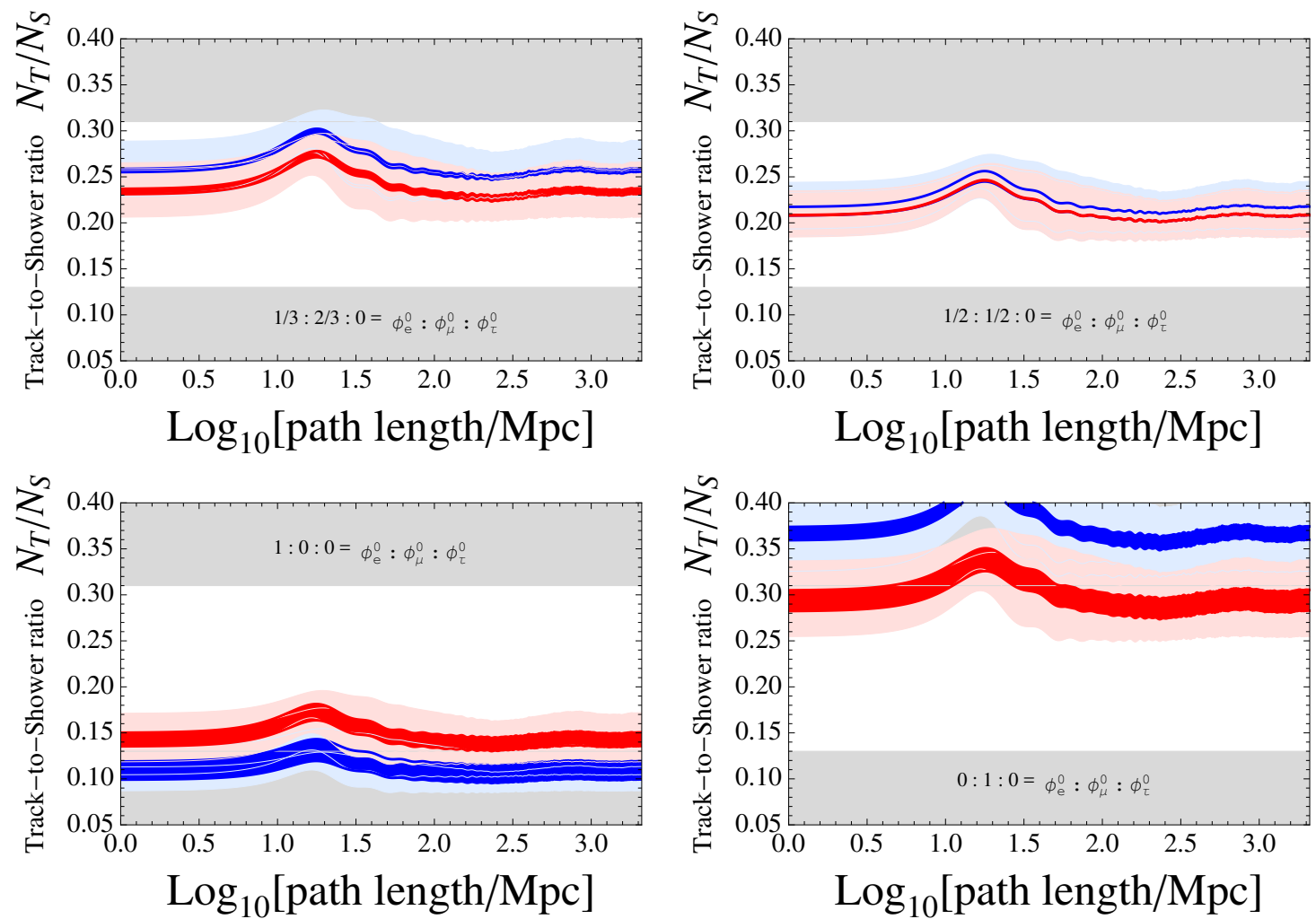

Figure 2. Plots of the track-to-shower ratio $N_{T} / N_{S}$ for normal (inverted) mass ordering as a function of $L\left(\log _{10}\right.$ [path length/Mpc]) for $\Delta m_{1,2,3}^{2}=10^{-16} \mathrm{eV}^{2}$. Each panel corresponds to the specific initial flavor composition $\left(\phi_{e}^{0}: \phi_{\mu}^{0}: \phi_{\tau}^{0}\right)$ at the source. For three neutrino mixing angles and Dirac-type CP phase, we take the global fit results at $1 \sigma$ [95]. Red and blue bands correspond to normal and inverted neutrino mass orderings, respectively, for $\alpha=2.2$, whereas light red and light blue regions represent the corresponding results for $\alpha=1.8-2.6$. Gray shaded regions represent the forbidden bound from $N_{T} / N_{S}=0.18_{-0.05}^{+0.13}$ in ref. [70].

\subsection{Results for the case of $\Delta m_{i}^{2} \gg \Delta m_{j}^{2}$}

In this case, we take $\Delta m_{1}^{2}=10^{-14} \mathrm{eV}^{2}, \Delta m_{2}^{2}=10^{-15} \mathrm{eV}^{2}$, and $\Delta m_{3}^{2}=10^{-16} \mathrm{eV}^{2}$ as a benchmark point. In the numerical analysis, we take the input values except for $\Delta m_{k}^{2}$ to be the same as in figure 2. In figures 3 and 4 , we plot the track-to-shower ratio $N_{T} / N_{S}$ as a function of $L\left(\log _{10}[\right.$ path length/Mpc] $)$ for the inverted and normal neutrino mass orderings, respectively. Initial flavor compositions at the source are the same as in figure 2. Gray shaded regions represent the forbidden bound from $N_{T} / N_{S}=0.18_{-0.05}^{+0.13}$ in ref. [70]. We can see from figure 3 that the results for the case with initial flavor composition $\left(\phi_{e}^{0}: \phi_{\mu}^{0}\right.$ : $\left.\phi_{\tau}^{0}\right)=(0: 1: 0)$ at the source is not consistent with experimental results, whereas only the highest region for the case with $\left(\phi_{e}^{0}: \phi_{\mu}^{0}: \phi_{\tau}^{0}\right)=(1: 0: 0)$ is consistent with experimental results. As can be seen from figure 4 , the predictions of $N_{T} / N_{S}$ for the normal mass ordering are all consistent with experimental results. Different from the results of figure 2 , the oscillation peaks in each panels in figures 3 and 4 are not so sharp. This means that the predictions of $N_{T} / N_{S}$ in these cases are deviated from the case with no new oscillatory effect for rather wider regions of the parameter $L$. 

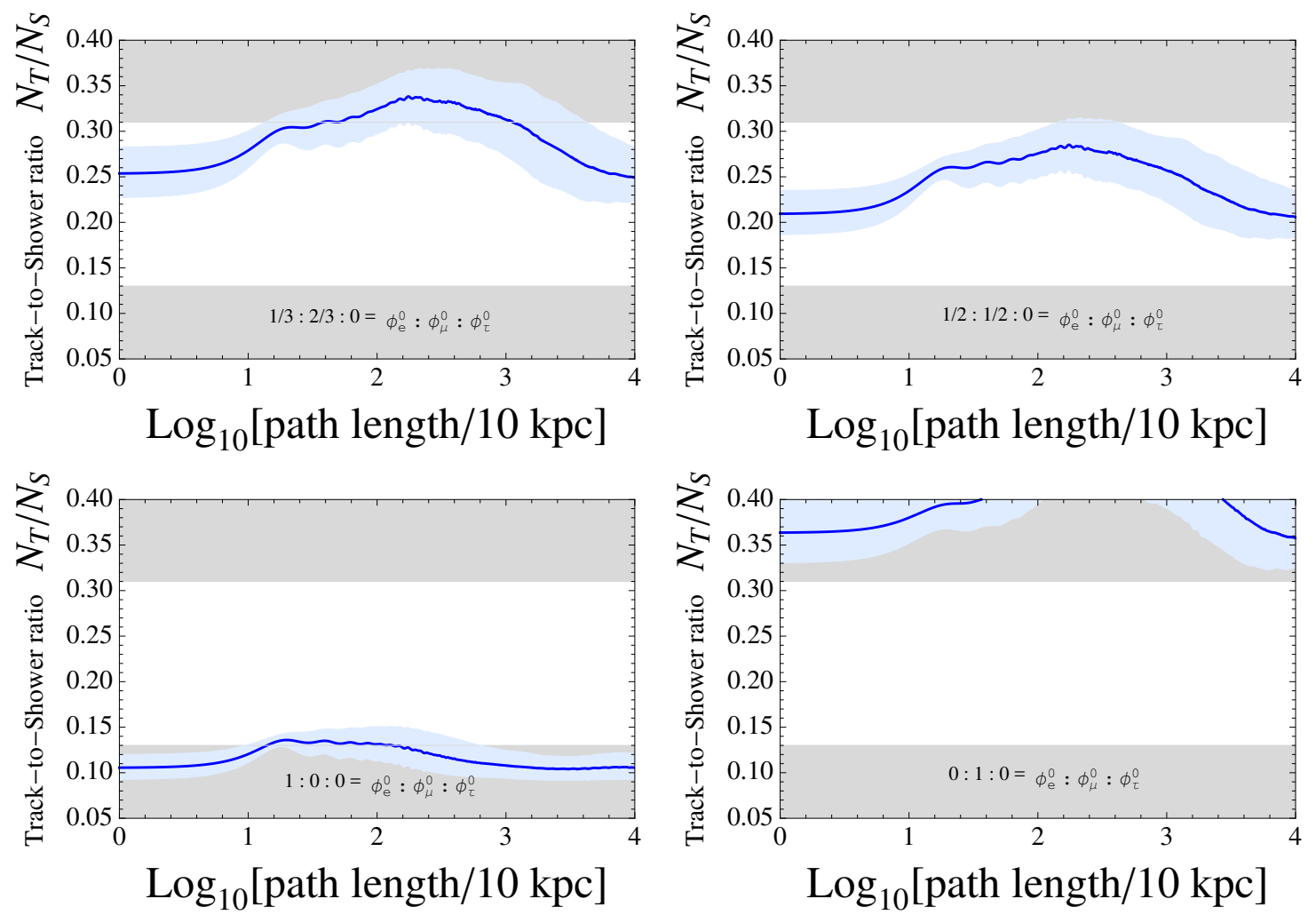

Figure 3. Plots of $N_{T} / N_{S}$ for inverted neutrino mass ordering as a function of $L\left(\log _{10}[\mathrm{path}\right.$ length/Mpc]) for $\Delta m_{1}^{2}=10^{-14} \mathrm{eV}^{2}, \Delta m_{2}^{2}=10^{-15} \mathrm{eV}^{2}$, and $\Delta m_{3}^{2}=10^{-16} \mathrm{eV}^{2}$. The input values except for $\Delta m_{k}^{2}$ are taken to be the same as in figure 2. Gray shaded regions represent the forbidden bound from $N_{T} / N_{S}=0.18_{-0.05}^{+0.13}$ in ref. [70]. Each panel corresponds to the specific initial flavor composition at the source, as in figure 2.

\begin{tabular}{|c|c|c|c|c|c|c|c|}
\hline$E(\mathrm{PeV})$ & $(0.523,0.627)$ & $(0.627,0.754)$ & $(0.754,0.905)$ & $(0.905,1.312)$ & $(1.312,1.576)$ & $(1.576,2,286)$ & $(2.286,3)$ \\
\hline $\operatorname{Dev}(\%)$ & 15.7 & 15.9 & 16.0 & 16.1 & 16.2 & 16.3 & 16.4 \\
\hline
\end{tabular}

Table 2. Deviation of the prediction for $N_{T} / N_{S}$ from the case without new oscillatory effect for $\Delta m_{k}^{2}=10^{-16} \mathrm{eV}^{2}$.

Up to this point, we have presented the numerical results for $N_{T} / N_{S}$ as a function of $L$ for a given $\Delta m_{k}^{2}$ and $60 \mathrm{TeV} \lesssim E \lesssim 3 \mathrm{PeV}$. However, the experimental results on high energy neutrinos released from IceCube are shown for narrow interval of energy from $60 \mathrm{TeV}$ to $3 \mathrm{PeV}$. From our numerical results, we see that the amount to which the prediction for $N_{T} / N_{S}$ is deviated from the one without new oscillatory effect depends on the energy scale. In table 2, we present how the prediction for $N_{T} / N_{S}$ is deviated from the case without new oscillatory effect for $\Delta m_{k}^{2}=10^{-16} \mathrm{eV}^{2}$ and $L(z)=1.1 \mathrm{Gpc}$. According to table 2, we see that the largest deviation occurs for $2.286 \lesssim E \lesssim 3 \mathrm{PeV}$. The deviation becomes smaller as $E$ goes lower. Similar result is obtained for the hierarchical case. Thus, more PeV scale data would be desirable to test our model.

In order to probe the presence of peudo-Dirac neutrino, observation of new oscillatory effects in $N_{T} / N_{S}$ is essential. To do this, future experiments should precisely measure the 

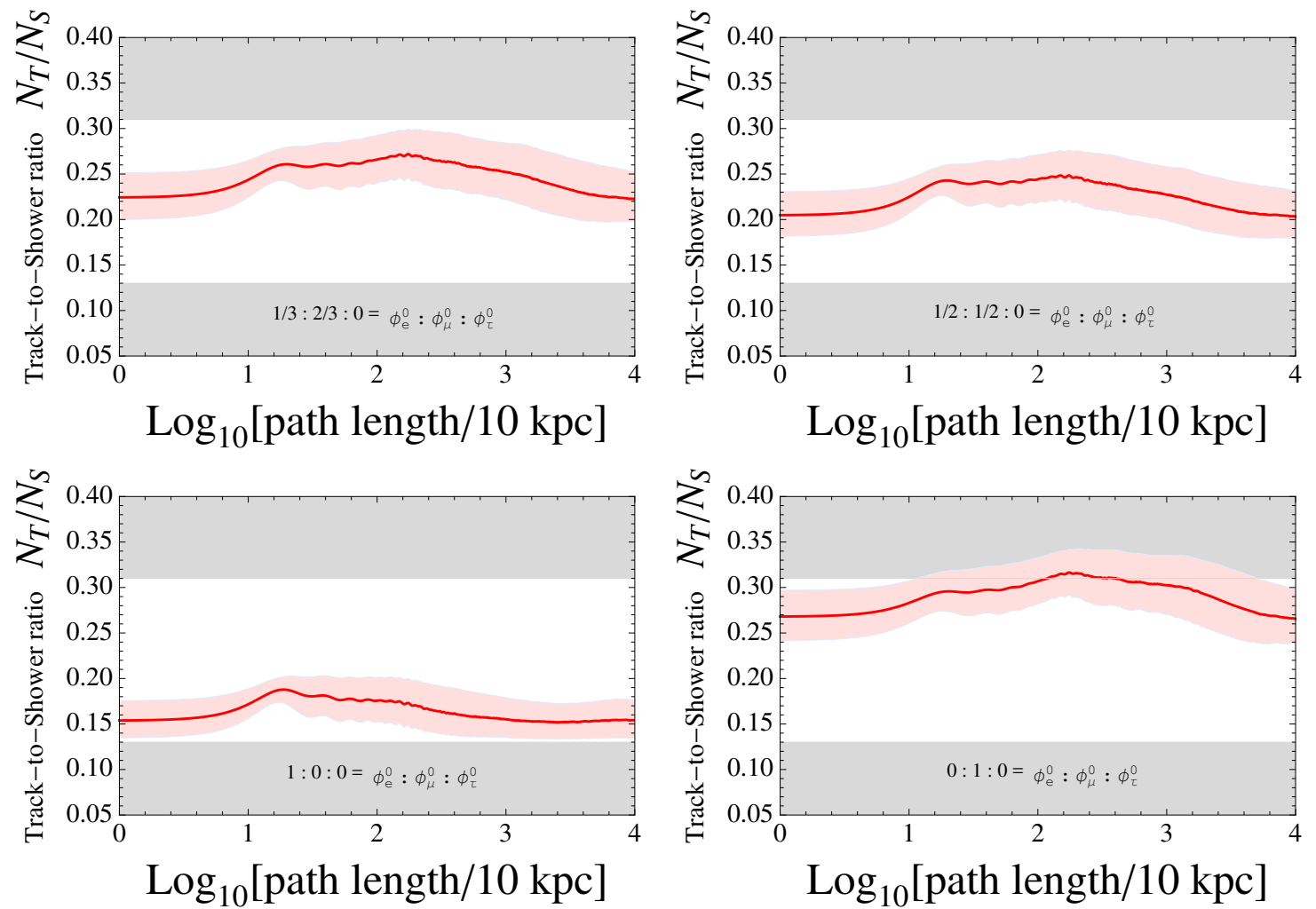

Figure 4. Plots of $N_{T} / N_{S}$ for normal neutrino mass ordering as a function of $L\left(\log _{10}[\mathrm{path}\right.$ length/Mpc]) for $\Delta m_{1}^{2}=10^{-14} \mathrm{eV}^{2}, \Delta m_{2}^{2}=10^{-15} \mathrm{eV}^{2}$, and $\Delta m_{3}^{2}=10^{-16} \mathrm{eV}^{2}$. The input values except for $\Delta m_{k}^{2}$ are taken to be the same as in 2. Gray shaded regions represent the forbidden bound from $N_{T} / N_{S}=0.18_{-0.05}^{+0.13}$ in ref. [70]. Each panel corresponds to the specific initial flavor composition at the source, as in figure 2 .

value of $N_{T} / N_{S}$. If the uncertainty in future measurements could be reduced by $50-60 \%$ from the current one without changing the central value, there would be a high chance to observe the new oscillatory effects via the oscillation peak for the case of degenerate $\Delta m_{k}^{2}$, and we would be able to test the pseudo-Dirac property of neutrinos, particularly for the cases with two flavors in the initial flavor composition of neutrino flux. For the case of hierarchical $\Delta m_{k}^{2}$, to test the model for peudo-Dirac neutrino, we need to reduce the uncertainty by $40-70 \%$ depending on the initial flavor compositions.

As expected, for $3 \sigma$ data of three neutrino oscillations one could not distinguish normal and inverted orderings for the track-to-shower ratio $N_{T} / N_{S}$, while the band width can be enlarged.

We can constrain mass squared differences $\Delta m_{1}^{2}$ and $\Delta m_{2}^{2}$ from the fact that the UHE neutrinos with energy $10^{9} \mathrm{GeV}$ are expected from the Greisen-Zatsepin-Kuzmin limit cosmic rays originated at distances of $100 \mathrm{Mpc}$ [97]. To observe such UHE neutrinos through neutrino oscillation whose length is of order $100 \mathrm{Mpc}$, the required magnitudes of $\delta_{1}$ and $\delta_{2}$ can be estimated from

$$
L_{\mathrm{osc}}^{1,2} \simeq\left(\frac{0.8 \times 10^{-12} \mathrm{eV}^{2}}{\Delta m_{1,2}^{2}}\right)\left(\frac{E}{10^{9} \mathrm{GeV}}\right) 100 \mathrm{Mpc} \lesssim 100 \mathrm{Mpc},
$$


which means that for such neutrinos with $E \sim 10^{9} \mathrm{GeV}$, oscillation length will be order of $100 \mathrm{Mpc}$ for $\Delta m_{1,2}^{2} \simeq 10^{-12} \mathrm{eV}^{2}$. In other words, $\delta_{1,2} \geq 4.6 \times 10^{-11} \mathrm{eV}$ (for normal mass hierarchy) and $\delta_{1,2} \geq 0.8 \times 10^{-11} \mathrm{eV}$ (for inverted mass hierarchy) are required for significant conversion of these neutrinos. Taking into account oscillation length of order the earth-sun distance 1 A.U. for neutrino energy $10^{9} \mathrm{GeV}$, we estimate the mass splittings are so large $\Delta m_{1,2}^{2} \simeq 16.6 \mathrm{eV}^{2}$ which contradicts with $\Delta m_{k}^{2} \ll \Delta m_{\text {sol }}^{2}$. Thereby, electron neutrinos from the nearby sources and high energy can remain undepleted, but ones from extragalactic sources get depleted.

\section{Conclusion}

In this work, we have proposed a model where sterile neutrinos are introduced to make light neutrinos to be pseudo-Dirac particles. It has been shown how tiny mass splitting necessary for realizing pseudo-Dirac neutrinos can be achieved. Within the model, we have examined how leptogenesis can be successfully generated. Motivated by the recent observation of very high energy neutrino events at IceCube and the results for the track-toshower ratio, $N_{T} / N_{S}$, of the subset with energy above $60 \mathrm{TeV}$ studied in ref. [70], we have examined a possibility to observe the effects of the pseudo-Dirac property of neutrinos by performing astronomical-scale baseline experiments to uncover the oscillation effects of very tiny mass splitting. Using the result of global fit to neutrino data for the input of neutrino mixing angles and CP phase at $1 \sigma$ C.L. and fixing neutrino energy and mass splittings, we have studied how the oscillation effects induced by pseudo-Dirac neutrinos may affect the track-to-shower ratio, and found that the oscillation peaks occur at the distance about $1.3 \mathrm{Gpc}$ for $\Delta m_{1,2,3}^{2}=10^{-16} \mathrm{eV}^{2}$ and $60 \mathrm{TeV} \lesssim E_{\nu} \lesssim 3 \mathrm{PeV}$. If future experiments can precisely measure the value of $N_{T} / N_{S}$, whose uncertainty becomes reduced to about $40-$ $70 \%$ depending on the initial flavor compositions at the source, we could test the pseudoDirac property of neutrinos particulary for the cases with two flavors in the initial flavor composition of neutrino flux. In fact, in order to obtain fully meaningful results for testing our model in detail, much larger detectors than the present IceCube would be required [98].

\section{Acknowledgments}

We woule like to thank Francis Halzen for valuable comments on the ultra-high energy cosmic neutrinos. The work of SKK is supported by NRF-2014R1A1A2057665. The work of Y.H. Ahn is supported by IBS under the project code, IBS-R018-D1. The work of CSK was supported by NRF grant funded by the Korea government of the MEST (No. 20110017430), (No. 2011-0020333).

Open Access. This article is distributed under the terms of the Creative Commons Attribution License (CC-BY 4.0), which permits any use, distribution and reproduction in any medium, provided the original author(s) and source are credited. 


\section{References}

[1] S. Dodelson and L.M. Widrow, Sterile-neutrinos as dark matter, Phys. Rev. Lett. 72 (1994) 17 [hep-ph/9303287] [INSPIRE].

[2] X.-D. Shi and G.M. Fuller, A New dark matter candidate: Nonthermal sterile neutrinos, Phys. Rev. Lett. 82 (1999) 2832 [astro-ph/9810076] [INSPIRE].

[3] A.D. Dolgov and S.H. Hansen, Massive sterile neutrinos as warm dark matter, Astropart. Phys. 16 (2002) 339 [hep-ph/0009083] [INSPIRE].

[4] K. Abazajian, G.M. Fuller and M. Patel, Sterile neutrino hot, warm and cold dark matter, Phys. Rev. D 64 (2001) 023501 [astro-ph/0101524] [INSPIRE].

[5] U. Seljak, A. Makarov, P. McDonald and H. Trac, Can sterile neutrinos be the dark matter?, Phys. Rev. Lett. 97 (2006) 191303 [astro-ph/0602430] [INSPIRE].

[6] A. Kusenko, Sterile neutrinos, dark matter and the pulsar velocities in models with a Higgs singlet, Phys. Rev. Lett. 97 (2006) 241301 [hep-ph/0609081] [INSPIRE].

[7] M. Laine and M. Shaposhnikov, Sterile neutrino dark matter as a consequence of

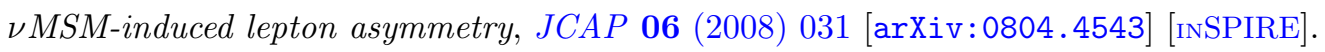

[8] K. Petraki and A. Kusenko, Dark-matter sterile neutrinos in models with a gauge singlet in the Higgs sector, Phys. Rev. D 77 (2008) 065014 [arXiv:0711.4646] [InSPIRE].

[9] S. Khalil and O. Seto, Sterile neutrino dark matter in B $-L$ extension of the standard model and galactic $511 \mathrm{keV}$ line, JCAP 10 (2008) 024 [arXiv:0804.0336] [INSPIRE].

[10] F. Bezrukov, H. Hettmansperger and M. Lindner, keV sterile neutrino Dark Matter in gauge extensions of the Standard Model, Phys. Rev. D 81 (2010) 085032 [arXiv:0912.4415] [INSPIRE].

[11] G.B. Gelmini, E. Osoba and S. Palomares-Ruiz, Inert-Sterile Neutrino: Cold or Warm Dark Matter Candidate, Phys. Rev. D 81 (2010) 063529 [arXiv:0912.2478] [INSPIRE].

[12] L. Canetti, M. Drewes and M. Shaposhnikov, Sterile Neutrinos as the Origin of Dark and Baryonic Matter, Phys. Rev. Lett. 110 (2013) 061801 [arXiv:1204.3902] [INSPIRE].

[13] P.S. Bhupal Dev and A. Pilaftsis, Light and Superlight Sterile Neutrinos in the Minimal Radiative Inverse Seesaw Model, Phys. Rev. D 87 (2013) 053007 [arXiv:1212.3808] [INSPIRE].

[14] A. Esmaili, S.K. Kang and P.D. Serpico, IceCube events and decaying dark matter: hints and constraints, JCAP 12 (2014) 054 [arXiv: 1410.5979] [INSPIRE].

[15] S.B. Roland, B. Shakya and J.D. Wells, Neutrino Masses and Sterile Neutrino Dark Matter from the PeV Scale, Phys. Rev. D 92 (2015) 113009 [arXiv:1412.4791] [InSPIRE].

[16] M. Frigerio and C.E. Yaguna, Sterile Neutrino Dark Matter and Low Scale Leptogenesis from a Charged Scalar, Eur. Phys. J. C 75 (2015) 31 [arXiv:1409.0659] [InSPIRE].

[17] K.C.Y. Ng, S. Horiuchi, J.M. Gaskins, M. Smith and R. Preece, Improved Limits on Sterile Neutrino Dark Matter using Full-Sky Fermi Gamma-Ray Burst Monitor Data, Phys. Rev. D 92 (2015) 043503 [arXiv: 1504.04027] [INSPIRE].

[18] A. Adulpravitchai and M.A. Schmidt, Sterile Neutrino Dark Matter Production in the Neutrino-phillic Two Higgs Doublet Model, JHEP 12 (2015) 023 [arXiv:1507.05694] [INSPIRE]. 
[19] M. Drewes and J.U. Kang, Sterile neutrino Dark Matter production from scalar decay in a thermal bath, JHEP 05 (2016) 051 [arXiv: 1510.05646] [INSPIRE].

[20] A. Merle, A. Schneider and M. Totzauer, Dodelson-Widrow Production of Sterile Neutrino Dark Matter with Non-Trivial Initial Abundance, JCAP 04 (2016) 003 [arXiv:1512.05369] [INSPIRE].

[21] B. Shakya, Sterile Neutrino Dark Matter from Freeze-In, Mod. Phys. Lett. A 31 (2016) 1630005 [arXiv: 1512.02751] [INSPIRE].

[22] T. Yanagida, Horizontal Symmetry And Masses Of Neutrinos, in proceedings of the Workshop on the Baryon Number of the Universe and Unified Theories, Tsukuba, Japan, February 13-14 1979, O. Sawada and A. Sugamoto eds., National Lab for High Energy Physics (1979) [Conf. Proc. C 7902131 (1979) 95] [INSPIRE].

[23] M. Gell-Mann, P. Ramond and R. Slansky, Complex spinors and unified theories, in Supergravity, P. Van Nieuwenhuizen and D.Z. Freeman eds., North Holland, Amsterdam The Netherlands (1979) [Conf. Proc. C 790927 (1979) 315] [arXiv:1306.4669] [inSPIRE].

[24] R.N. Mohapatra and G. Senjanović, Neutrino Mass and Spontaneous Parity Violation, Phys. Rev. Lett. 44 (1980) 912 [INSPIRE].

[25] M. Fukugita and T. Yanagida, Baryogenesis Without Grand Unification, Phys. Lett. B 174 (1986) 45 [INSPIRE].

[26] C.O. Dib and C.S. Kim, Discovering sterile Neutrinos ligther than $M_{W}$ at the LHC, Phys. Rev. D 92 (2015) 093009 [arXiv:1509.05981] [InSPIRE].

[27] F.F. Deppisch, P.S. Bhupal Dev and A. Pilaftsis, Neutrinos and Collider Physics, New J. Phys. 17 (2015) 075019 [arXiv: 1502.06541] [INSPIRE].

[28] S. Antusch, E. Cazzato and O. Fischer, Higgs production from sterile neutrinos at future lepton colliders, JHEP 04 (2016) 189 [arXiv: 1512.06035] [INSPIRE].

[29] A. Kusenko, Sterile neutrinos: The Dark side of the light fermions, Phys. Rept. 481 (2009) 1 [arXiv: 0906.2968] [INSPIRE].

[30] L. Basso, O. Fischer and J.J. van der Bij, Precision tests of unitarity in leptonic mixing, Europhys. Lett. 105 (2014) 11001 [arXiv:1310. 2057] [INSPIRE].

[31] S. Antusch and O. Fischer, Non-unitarity of the leptonic mixing matrix: Present bounds and future sensitivities, JHEP 10 (2014) 094 [arXiv: 1407.6607] [INSPIRE].

[32] C. Giunti, Light Sterile Neutrinos: Status and Perspectives, Nucl. Phys. B 908 (2016) 336 [arXiv: 1512.04758] [INSPIRE].

[33] S. Gariazzo, C. Giunti, M. Laveder, Y.F. Li and E.M. Zavanin, Light sterile neutrinos, J. Phys. G 43 (2016) 033001 [arXiv: 1507.08204] [InSPIRE].

[34] E.J. Konopinski and H.M. Mahmoud, The Universal Fermi interaction, Phys. Rev. 92 (1953) 1045 [INSPIRE].

[35] L. Wolfenstein, Different Varieties of Massive Dirac Neutrinos, Nucl. Phys. B 186 (1981) 147 [INSPIRE].

[36] C. Giunti, C.W. Kim and U.W. Lee, Oscillations of pseudo Dirac neutrinos and the solar neutrino problem, Phys. Rev. D 46 (1992) 3034 [hep-ph/9205214] [INSPIRE].

[37] A. Geiser, Pseudo-Dirac neutrinos as a potential complete solution to the neutrino oscillation puzzle, Phys. Lett. B 444 (1999) 358 [hep-ph/9901433] [InSPIRE]. 
[38] W. Krolikowski, Option of three pseudo-Dirac neutrinos, Acta Phys. Polon. B 31 (2000) 663 [hep-ph/9910308] [INSPIRE].

[39] D. Chang and O.C.W. Kong, Pseudo-Dirac neutrinos, Phys. Lett. B 477 (2000) 416 [hep-ph/9912268] [INSPIRE].

[40] Y. Nir, Pseudo-Dirac solar neutrinos, JHEP 06 (2000) 039 [hep-ph/0002168] [INSPIRE].

[41] A.S. Joshipura and S.D. Rindani, Phenomenology of pseudo Dirac neutrinos, Phys. Lett. B 494 (2000) 114 [hep-ph/0007334] [INSPIRE].

[42] K.R.S. Balaji, A. Kalliomaki and J. Maalampi, Revisiting pseudo-Dirac neutrinos, Phys. Lett. B 524 (2002) 153 [hep-ph/0110314] [INSPIRE].

[43] J.F. Beacom, N.F. Bell, D. Hooper, J.G. Learned, S. Pakvasa and T.J. Weiler, Pseudo-Dirac neutrinos: A Challenge for neutrino telescopes, Phys. Rev. Lett. 92 (2004) 011101 [hep-ph/0307151] [INSPIRE].

[44] A. de Gouvêa, W.-C. Huang and J. Jenkins, Pseudo-Dirac Neutrinos in the New Standard Model, Phys. Rev. D 80 (2009) 073007 [arXiv:0906.1611] [InSPIRE].

[45] A.S. Joshipura, S. Mohanty and S. Pakvasa, Pseudo-Dirac neutrinos via a mirror world and depletion of ultrahigh energy neutrinos, Phys. Rev. D 89 (2014) 033003 [arXiv:1307.5712] [INSPIRE].

[46] A. Esmaili and Y. Farzan, Implications of the Pseudo-Dirac Scenario for Ultra High Energy Neutrinos from GRBs, JCAP 12 (2012) 014 [arXiv:1208.6012] [INSPIRE].

[47] A. Esmaili, Pseudo-Dirac Neutrino Scenario: Cosmic Neutrinos at Neutrino Telescopes, Phys. Rev. D 81 (2010) 013006 [arXiv:0909.5410] [InSPIRE].

[48] Planck collaboration, P.A.R. Ade et al., Planck 2015 results. XIII. Cosmological parameters, Astron. Astrophys. 594 (2016) A13 [arXiv: 1502.01589] [INSPIRE].

[49] Particle Data Group collaboration, K.A. Olive et al., Review of Particle Physics, Chin. Phys. C 38 (2014) 090001 [inSPIRE].

[50] C. Giunti and C.W. Kim, Fundamentals of neutrinno Physics and Astrophysics, Oxford University Press, Oxpord U.K. (2007) [ISBN: 978-0-19-850871-7].

[51] G. Barenboim and C. Quigg, Neutrino observatories can characterize cosmic sources and neutrino properties, Phys. Rev. D 67 (2003) 073024 [hep-ph/0301220] [INSPIRE].

[52] C. Quigg, Extremely high energy cosmic neutrinos and relic neutrinos, astro-ph/0603372 [INSPIRE].

[53] J.F. Beacom, N.F. Bell, D. Hooper, S. Pakvasa and T.J. Weiler, Measuring flavor ratios of high-energy astrophysical neutrinos, Phys. Rev. D 68 (2003) 093005 [Erratum ibid. D 72 (2005) 019901] [hep-ph/0307025] [INSPIRE].

[54] Z.-Z. Xing and S. Zhou, Towards determination of the initial flavor composition of ultrahigh-energy neutrino fluxes with neutrino telescopes, Phys. Rev. D 74 (2006) 013010 [astro-ph/0603781] [INSPIRE].

[55] W. Winter, How astrophysical neutrino sources could be used for early measurements of neutrino mass hierarchy and leptonic CP phase, Phys. Rev. D 74 (2006) 033015 [hep-ph/0604191] [INSPIRE].

[56] S. Pakvasa, W. Rodejohann and T.J. Weiler, Flavor Ratios of Astrophysical Neutrinos: Implications for Precision Measurements, JHEP 02 (2008) 005 [arXiv:0711.4517] [INSPIRE]. 
[57] A. Esmaili and Y. Farzan, An Analysis of Cosmic Neutrinos: Flavor Composition at Source and Neutrino Mixing Parameters, Nucl. Phys. B 821 (2009) 197 [arXiv:0905.0259] [INSPIRE].

[58] K.-C. Lai, G.-L. Lin and T.C. Liu, Determination of the Neutrino Flavor Ratio at the Astrophysical Source, Phys. Rev. D 80 (2009) 103005 [arXiv:0905.4003] [INSPIRE].

[59] S. Choubey and W. Rodejohann, Flavor Composition of UHE Neutrinos at Source and at Neutrino Telescopes, Phys. Rev. D 80 (2009) 113006 [arXiv: 0909.1219] [InSPIRE].

[60] P. Mehta and W. Winter, Interplay of energy dependent astrophysical neutrino flavor ratios and new physics effects, JCAP 03 (2011) 041 [arXiv:1101.2673] [INSPIRE].

[61] A. Chatterjee, M.M. Devi, M. Ghosh, R. Moharana and S.K. Raut, Probing CP-violation with the first three years of ultrahigh energy neutrinos from IceCube, Phys. Rev. D 90 (2014) 073003 [arXiv: 1312.6593] [INSPIRE].

[62] O. Mena, S. Palomares-Ruiz and A.C. Vincent, Flavor Composition of the High-Energy Neutrino Events in IceCube, Phys. Rev. Lett. 113 (2014) 091103 [arXiv:1404.0017] [INSPIRE].

[63] C.-Y. Chen, P.S. Bhupal Dev and A. Soni, Two-component flux explanation for the high energy neutrino events at IceCube, Phys. Rev. D 92 (2015) 073001 [arXiv:1411.5658] [INSPIRE].

[64] X.-J. Xu, H.-J. He and W. Rodejohann, Constraining Astrophysical Neutrino Flavor Composition from Leptonic Unitarity, JCAP 12 (2014) 039 [arXiv:1407.3736] [INSPIRE].

[65] S. Palomares-Ruiz, A.C. Vincent and O. Mena, Spectral analysis of the high-energy IceCube neutrinos, Phys. Rev. D 91 (2015) 103008 [arXiv:1502.02649] [INSPIRE].

[66] M. Bustamante, J.F. Beacom and W. Winter, Theoretically palatable flavor combinations of astrophysical neutrinos, Phys. Rev. Lett. 115 (2015) 161302 [arXiv:1506.02645] [INSPIRE].

[67] I.M. Shoemaker and K. Murase, Probing BSM Neutrino Physics with Flavor and Spectral Distortions: Prospects for Future High-Energy Neutrino Telescopes, Phys. Rev. D 93 (2016) 085004 [arXiv: 1512.07228] [INSPIRE].

[68] IceCube collaboration, M.G. Aartsen et al., Observation of High-Energy Astrophysical Neutrinos in Three Years of IceCube Data, Phys. Rev. Lett. 113 (2014) 101101 [arXiv: 1405.5303] [INSPIRE].

[69] ICECUBe collaboration, M.G. Aartsen et al., Flavor Ratio of Astrophysical Neutrinos above 35 TeV in IceCube, Phys. Rev. Lett. 114 (2015) 171102 [arXiv:1502.03376] [INSPIRE].

[70] A. Palladino, G. Pagliaroli, F.L. Villante and F. Vissani, What is the Flavor of the Cosmic Neutrinos Seen by IceCube?, Phys. Rev. Lett. 114 (2015) 171101 [arXiv:1502.02923] [INSPIRE].

[71] M. Kobayashi and C.S. Lim, Pseudo Dirac scenario for neutrino oscillations, Phys. Rev. D 64 (2001) 013003 [hep-ph/0012266] [INSPIRE].

[72] S. Blanchet, P.S.B. Dev and R.N. Mohapatra, Leptogenesis with TeV Scale Inverse Seesaw in S0(10), Phys. Rev. D 82 (2010) 115025 [arXiv:1010.1471] [INSPIRE].

[73] L. Basso, O. Fischer and J.J. van der Bij, Natural $Z^{\prime}$ model with an inverse seesaw mechanism and leptonic dark matter, Phys. Rev. D 87 (2013) 035015 [arXiv:1207.3250] [INSPIRE]. 
[74] W. Buchmüller, R.D. Peccei and T. Yanagida, Leptogenesis as the origin of matter, Ann. Rev. Nucl. Part. Sci. 55 (2005) 311 [hep-ph/0502169] [INSPIRE].

[75] G.F. Giudice, A. Notari, M. Raidal, A. Riotto and A. Strumia, Towards a complete theory of thermal leptogenesis in the SM and MSSM, Nucl. Phys. B 685 (2004) 89 [hep-ph/0310123] [INSPIRE].

[76] WMAP collaboration, C.L. Bennett et al., Nine-Year Wilkinson Microwave Anisotropy Probe (WMAP) Observations: Final Maps and Results, Astrophys. J. Suppl. 208 (2013) 20 [arXiv: 1212.5225] [INSPIRE].

[77] WMAP collaboration, G. Hinshaw et al., Nine-Year Wilkinson Microwave Anisotropy Probe (WMAP) Observations: Cosmological Parameter Results, Astrophys. J. Suppl. 208 (2013) 19 [arXiv: 1212.5226] [INSPIRE].

[78] A. Pilaftsis, CP violation and baryogenesis due to heavy Majorana neutrinos, Phys. Rev. D 56 (1997) 5431 [hep-ph/9707235] [INSPIRE].

[79] M. Kawasaki, K. Kohri and T. Moroi, Big-Bang nucleosynthesis and hadronic decay of long-lived massive particles, Phys. Rev. D 71 (2005) 083502 [astro-ph/0408426] [INSPIRE].

[80] M.Y. Khlopov and A.D. Linde, Is It Easy to Save the Gravitino?, Phys. Lett. B 138 (1984) 265 [INSPIRE].

[81] S.K. Kang and C.S. Kim, Extended double seesaw model for neutrino mass spectrum and low scale leptogenesis, Phys. Lett. B 646 (2007) 248 [hep-ph/0607072] [INSPIRE].

[82] S.K. Kang and C.S. Kim, Extended double seesaw model and low scale leptogenesis, Int. J. Mod. Phys. E 16 (2007) 1505 [INSPIRE].

[83] H. Sung Cheon, S.K. Kang and C.S. Kim, Low Scale Leptogenesis and Dark Matter Candidates in an Extended Seesaw Model, JCAP 05 (2008) 004 [Erratum ibid. 03 (2011) E01] [arXiv:0710.2416] [INSPIRE].

[84] S.K. Kang and A. Patra, keV Sterile Neutrino Dark Matter and Low Scale Leptogenesis, arXiv: 1412.4899 [INSPIRE].

[85] E. Waxman and J.N. Bahcall, High-energy neutrinos from cosmological gamma-ray burst fireballs, Phys. Rev. Lett. 78 (1997) 2292 [astro-ph/9701231] [INSPIRE].

[86] E. Waxman and J.N. Bahcall, High-energy neutrinos from astrophysical sources: An Upper bound, Phys. Rev. D 59 (1999) 023002 [hep-ph/9807282] [InSPIRE].

[87] J.K. Becker, High-energy neutrinos in the context of multimessenger physics, Phys. Rept. 458 (2008) 173 [arXiv:0710.1557] [INSPIRE].

[88] K. Mannheim, R.J. Protheroe and J.P. Rachen, On the cosmic ray bound for models of extragalactic neutrino production, Phys. Rev. D 63 (2001) 023003 [astro-ph/9812398] [INSPIRE].

[89] K. Mannheim, High-energy neutrinos from extragalactic jets, Astropart. Phys. 3 (1995) 295 [INSPIRE].

[90] A. Kappes, J. Hinton, C. Stegmann and F.A. Aharonian, Potential Neutrino Signals from Galactic Gamma-Ray Sources, Astrophys. J. 656 (2007) 870 [Erratum ibid. 661 (2007) 1348] [astro-ph/0607286] [INSPIRE].

[91] S. Gabici and F.A. Aharonian, Searching for galactic cosmic ray pevatrons with multi-TeV gamma rays and neutrinos, Astrophys. J. 665 (2007) L131 [arXiv:0705.3011] [INSPIRE]. 
[92] F. Halzen, A. Kappes and A. O'Murchadha, Prospects for identifying the sources of the Galactic cosmic rays with IceCube, Phys. Rev. D 78 (2008) 063004 [arXiv:0803.0314] [INSPIRE].

[93] F. Vissani, Neutrinos from galactic sources of cosmic rays with known $\gamma$-ray spectra, Astropart. Phys. 26 (2006) 310 [astro-ph/0607249] [INSPIRE].

[94] C. Lunardini and A.Y. Smirnov, The Minimum width condition for neutrino conversion in matter, Nucl. Phys. B 583 (2000) 260 [hep-ph/0002152] [INSPIRE].

[95] M.C. Gonzalez-Garcia, M. Maltoni and T. Schwetz, Global Analyses of Neutrino Oscillation Experiments, Nucl. Phys. B 908 (2016) 199 [arXiv:1512.06856] [InSPIRE].

[96] ICECuBE collaboration, M.G. Aartsen et al., Evidence for High-Energy Extraterrestrial Neutrinos at the IceCube Detector, Science 342 (2013) 1242856 [arXiv:1311.5238] [INSPIRE].

[97] Pierre Auger collaboration, J. Abraham et al., Correlation of the highest energy cosmic rays with nearby extragalactic objects, Science 318 (2007) 938 [arXiv:0711.2256] [INSPIRE].

[98] IceCube collaboration, M.G. Aartsen et al., IceCube-Gen2: A Vision for the Future of Neutrino Astronomy in Antarctica, arXiv:1412.5106 [INSPIRE]. 